\title{
Does circular polarisation reveal the rotation of quasar engines?
}

\author{
T. A. Enßlin ${ }^{\star}$ \\ Max-Planck-Institut für Astrophysik, Karl-Schwarzschild-Str. 1, Postfach 1317, 85741 Garching, Germany \\ Received 6 November 2002 / Accepted 30 January 2003 \begin{abstract}
surprising temporal persistent handedness. As a possible explanation we propose that the $\mathrm{CP}$ is due to Faraday conversion (FC) of linear polarisation (LP) synchrotron light which propagates along a line-of-sight (LOS) through twisted magnetic fields. The rotational nature of accretion flows onto black holes naturally generates the required magnetic twist in the emission region, independent of whether it is a jet or an advection dominated accretion flow (ADAF). The expected twist in both types of flows is of the order of what is required for optimal CP generation. This scenario requires that Faraday rotation (FR) is insignificant scenarios which rely on FR, since there the strength of FR can not be too far from the optimum. The proposed mechanism works in electron-positron $\left(\mathrm{e}^{ \pm}\right)$as well as electron-proton (e/p) plasma. In the latter case, the emission region should consist of individual flux tubes with independent polarities in order to suppress too strong FR. The predominant CP is expected to mostly counter-rotate (rotation is measured here in sky-projection) with respect to the central engine in all cases (jet or ADAF, $\mathrm{e}^{ \pm}$or $\mathrm{e} / \mathrm{p}$ plasma). If the proposed mechanism is indeed operating, it will allow to measure the sense of rotation of quasar engines. The engine of SgrA* is then expected to rotate clockwise and therefore counter-Galactic, as do the young hot stars in its vicinity, which are thought to feed SgrA* by their winds. Similarly, we expect the microquasars SS 443 and GRS $1915+105$ to rotate clockwise. Generally, sources with Stokes- $V<0(V>0)$ are expected to rotate clockwise (counter-clockwise) in this scenario.
\end{abstract} \\ Abstract. Many radio sources like quasars, blazars, radio galaxies, and micro-quasars exhibit circular polarisation (CP) with \\ in the emission region. Although this is an assumption, it relaxes constraints on the plasma parameters, that were given in
}

Key words. radiation mechanisms: non-thermal - radio continuum: general - polarization - galaxies: active galaxies: jets - galaxies: magnetic fields

\section{Introduction}

The long lasting interest in $\mathrm{CP}$ from quasar-like systems (e.g. Jones \& O'Dell 1977a, in the following J\&O'D) increased recently due to the detection of several new CP sources as SgrA* (Bower et al. 1999; Sault \& Macquart 1999), M 81* (Brunthaler et al. 2001), the micro-quasars SS 433 and GRS 1915+105 (Fender et al. 2000, 2002), low luminosity AGNs (Bower et al. 2002a), and many additional powerful quasars (Wardle et al. 1998; Homan \& Wardle 1999) and blazars (Homan et al. 2001). In the following we will summarise these systems under the general term quasar, assuming that a similar mechanism produces CP in most of them. The level of CP is $\sim 1 \%$ and below, usually (but not in the case of $\operatorname{SgrA}^{*}$ ) much lower than the level of LP. On the one hand CP is highly time variable, but on the other hand in many sources it exhibits a very persistent rotational sense (per source and at nearly all frequencies where detected), which is constant on timescales of decades (Komesaroff et al. 1984; Homan \& Wardle 1999; Bower et al. 2002b), although exceptions exist. This is far in excess of the dynamical timescale of the central quasar engine from which the emission originates.

In these sources synchrotron radiation from relativistic electrons produces LP, but only a very small and usually

\footnotetext{
* e-mail: ensslin@mpa-garching.mpg.de
}

negligible amount of CP. A number of mechanisms have been proposed to explain the observed CP, among which FC of LP to $\mathrm{CP}$ seems to be the most likely process (Fender et al. 2002; Macquart et al. 2002). Beckert \& Falcke (2002) and Ruszkowski \& Begelman (2002) (B\&F and R\&B in the following) provide a good introductions into the matter, a detailed discussion of the various $\mathrm{CP}$ generation mechanisms, and further references. For a discussion of scintillation models for $\mathrm{CP}$ generation Macquart (2002) should be consulted.

FC of synchrotron emission is a two-step process, since the emitted polarisation state has to be rotated before it can be Faraday converted into circular polarisation. This can happen by FR, as discussed by e.g. B\&F and R\&B and used as a starting point of this work, or it can be done by a systematic geometrical rotation of the magnetic field along the LOS, as originally proposed by Hodge (1982) and discussed here.

The motivation for the standpoint adopted in this paper, the assumption that the rotation of LP is a geometric effect rather than $\mathrm{FR}$, is the observational fact that $\mathrm{CP}$ changes are very rare, much more rare than LP rotator events, and that a predominant $\mathrm{CP}$ sign seems to be persistent in many sources. As explained in the following, this observational fact requires in the FR based models a constant magnetic polarity in the emission region over timescales far in excess of the dynamical timescale of the central engine, since the sign of $\mathrm{CP}$ depends on the magnetic field 
polarity in such models. In the geometrical model discussed here, the CP sign is fully determined by the rotational sense of the central engine. Therefore the persistence of the predominant CP sign would - in this picture - be a natural consequence of angular momentum conservation.

\section{Faraday conversion}

\subsection{Homogeneous magnetic fields}

We start our discussion with the case of a homogeneous magnetic field, and follow the notation of J\&O'D, B\&F, and R\&B, which should be consulted for details and references. The evolution of the Stokes polarisation parameters $I, Q, U, V$ along a given LOS (here defining the $z$-axis) is governed by

$$
\frac{\mathrm{d}}{\mathrm{d} z}\left(\begin{array}{c}
I \\
Q \\
U \\
V
\end{array}\right)=\left(\begin{array}{c}
\varepsilon_{I} \\
\varepsilon_{Q} \\
0 \\
\varepsilon_{V}
\end{array}\right)-\left(\begin{array}{cccc}
\kappa_{I} & \kappa_{Q} & 0 & \kappa_{V} \\
\kappa_{Q} & \kappa_{I} & \kappa_{\mathrm{F}} & 0 \\
0 & -\kappa_{\mathrm{F}} & \kappa_{I} & \kappa_{\mathrm{C}} \\
\kappa_{V} & 0 & -\kappa_{\mathrm{C}} & \kappa_{I}
\end{array}\right)\left(\begin{array}{c}
I \\
Q \\
U \\
V
\end{array}\right),
$$

where $\varepsilon_{I / Q / U / V}$ is the emissivity and $\kappa_{I / Q / U / V}$ the absorption coefficient of radiation with Stokes parameter $I / Q / U / V$. A special coordinate frame is adopted, in which the sky-projected magnetic field is parallel to the $y$-axis $\left(\boldsymbol{B}=\left(0, B_{y}, B_{z}\right)\right)$, which leads to vanishing synchrotron emission and absorption coefficients for Stokes- $U^{1}$. The emission and absorption coefficients for Stokes- $V^{2}$ are much smaller than the ones for Stokes- $Q^{3}-$ if they are not also zero as would be the case for an $\mathrm{e}^{ \pm}$plasma $^{4}$. The FR coefficient reads

$\kappa_{\mathrm{F}}=-\frac{\tilde{\rho}_{\mathrm{e}} e^{2} B_{z}}{\pi m_{\mathrm{e}}^{2} c^{4}} \lambda^{2}$,

where $\tilde{\rho}_{\mathrm{e}}$ gives the effective Faraday-active leptonic charge density. For a non-relativistic plasma $\tilde{\rho}_{\mathrm{e}}$ is identical to the leptonic charge density $\rho_{\mathrm{e}}=e\left(n_{\mathrm{e}^{+}}-n_{\mathrm{e}^{-}}\right)$, and for a relativistic plasma $\tilde{\rho}_{\mathrm{e}} \propto \rho_{\mathrm{e}}$ with a constant of proportionality smaller than one $^{5}$. The sign of FR depends on the sign of $\rho_{\mathrm{e}}$ and on the direction of the magnetic field component along the LOS $\left(B_{z}\right)$. The FC coefficient

$\kappa_{\mathrm{C}}=-\frac{\tilde{n}_{\mathrm{e}} e^{4} B_{y}^{2}}{4 \pi^{2} m_{\mathrm{e}}^{3} c^{6}} \lambda^{3}$

is sensitive to the total number of leptonic charge carriers $\tilde{n}_{\mathrm{e}} \propto n_{\mathrm{e}}=n_{\mathrm{e}^{+}}+n_{\mathrm{e}^{-}}$(with a proportionality constant of one in the non-relativistic and smaller than one in the relativistic

\footnotetext{
${ }^{1} \mathrm{LP}, \pi / 4$ inclined to the sky-projected magnetic fields.

${ }^{2} \mathrm{CP}$, clockwise or negatively rotating in sky-projection for $V>0$, counter-clockwise or positively rotating for $V<0$.

${ }^{3}$ LP, perpendicular $(Q>0)$ or parallel $(Q<0)$ electric oscillation with respect to the sky-projected magnetic field.

4 Valtaoja (1984) proposed a model in which relativistic beaming allows to see the small amount of circularly polarised synchrotron emission, whereas the linear polarisation is suppressed by cancelling in a helical magnetic field configuration seen face on. Note, that in this model the CP sign depends on the magnetic polarity.

${ }^{5} n_{\mathrm{e}^{ \pm}}$is the $\mathrm{e}^{ \pm}$number density respectively. In the relativistic case an identical spectral distribution of the two species is assumed, otherwise they enter $\tilde{\rho}_{\mathrm{e}}$ with different proportionality factors.
}

case) and the square of the perpendicular field strength $B_{y}^{2}$. Since both numbers are always positive, $\kappa_{C}$ is always negative (or zero). Since both Faraday coefficients strongly increase with wavelength their importance is largest at lowest frequencies, possibly explaining why $\mathrm{CP}$ is often detected close to the synchrotron-self-absorption frequency. The relatively flat observed $V$ spectrum of $\mathrm{SgrA}^{*}$ (compared to the $I$ spectrum, Bower et al. 2002b) may indicate that for too strongly selfabsorbed sources CP production becomes inefficient, since it is expected to happen only within the $\tau=\int \mathrm{d} z \kappa_{I} \leq 1$ region. In an e/p plasma with diagonal magnetic fields $\left(\left|B_{y}\right| \sim\left|B_{z}\right|\right)$ the FR coefficient is usually larger in magnitude compared to the FC coefficient, except for extremely high field strength or extremely low frequencies. Both are not expected in our case.

Since there is no direct conversion of $Q$ synchrotron emission to $V$ (the corresponding matrix element in Eq. (1) is zero) $\mathrm{CP}$ has to be produced via a two-step conversion in this setting: after FR of $Q$ into $U$ it is further converted by $\mathrm{FC}$ to $V$. The sign of $V$ depends only on the sign of $\kappa_{\mathrm{F}}$ and therefore on the polarity of the magnetic field with respect to the LOS. In a charge symmetric $\mathrm{e}^{ \pm}$plasma $\left(\rho_{\mathrm{e}^{ \pm}}=0\right)$ FR vanishes, and this mechanism is unable to operate. On the other hand the total Faraday depth $\left(\tau_{\mathrm{F}}=\int \mathrm{d} z \kappa_{\mathrm{F}}\right)$ should not be too large within the converting region, otherwise the continued FR changes the sign of $U$, which leads to the production of $V$ with the opposite sign and therefore cancelling of $\mathrm{CP}$. An effective $\mathrm{CP}$ production can be achieved if both $\tau_{\mathrm{F}}$ and $\tau_{\mathrm{C}}=\int \mathrm{d} z \kappa_{\mathrm{C}}$ are simultaneously of the order one, since $V \approx \frac{1}{6} \tau_{\mathrm{F}} \tau_{\mathrm{C}} Q$ for $\tau_{\mathrm{F}} \ll 1$ and $\tau_{\mathrm{C}} \ll 1$ within an optically thin region ${ }^{6}$, and much less than this if $\tau_{\mathrm{F}} \geq 1$ and/or $\tau_{\mathrm{C}} \geq 1$ due to over-rotation and/or over-conversion.

In order to produce a significant $V$ component in the case of a homogenous magnetic field either $\kappa_{\mathrm{F}}$ has to be fine tuned with the help of a suitable field orientation ${ }^{7}$ or by a small but non-zero effective leptonic charge density, since $\kappa_{\mathrm{F}} / \kappa_{\mathrm{C}} \propto \varrho_{\mathrm{e}} / n_{\mathrm{e}}$. Both possibilities seem to be too contrived to allow $\mathrm{CP}$ to be detected in a significant number of very different sources, ranging from micro-quasars over low-luminosity AGNs to powerful quasars.

\subsection{Stochastic magnetic fields}

$\mathrm{B} \& \mathrm{~F}$ and $\mathrm{R} \& \mathrm{~B}$ demonstrated that a significant $\mathrm{CP}$ production can be achieved even in the case of a very large microscopic FR coefficient if the magnetic field is mostly stochastic.

\footnotetext{
${ }^{6}$ For an optically thick source, using only the region up to $\tau=1$ is a reasonable approximation, leading to $V \approx \frac{1}{6} \tau_{\mathrm{F}} \tau_{\mathrm{C}} Q / \tau^{2}$. CP sign changes can occur around $\tau \sim 1$ (Jones \& O'Dell 1977a, 1977b). However, the intensity of the CP flux with reversed sign is usually much below the flux with the predominant sign. For that reason Björnsson (1990) concluded that in inhomogeneous situations such $\mathrm{CP}$ reversals are usually suppressed. Anyhow, here we concentrate on the predominant sign of $\mathrm{CP}$, and ignore possible complication which can occur occasionally at special frequencies.

${ }^{7}$ Ideally, the field would be oriented mostly perpendicular to the LOS - but not perfectly, as B\&F point out by emphasising the advantage of a helical field configuration.
} 
The contributions of $\kappa_{\mathrm{F}}$ with differing signs due to magnetic field reversals can cancel each other, leaving only a small total Faraday depth $\tau_{\mathrm{F}}$ due to an assumed weak mean field. The strength and sign of this mean field determines the strength and sign of the resulting CP respectively. Although some finetuning is also necessary in this scenario in order to simultaneously have the right order of FR and FC, the larger number of free parameters (field strength and coherence length, ratio of mean to stochastic field components, $\tilde{\rho}_{\mathrm{e}}, \tilde{n}_{\mathrm{e}}$ ) provides a sufficiently large parameter space to make this scenario a plausible explanation for the observed $\mathrm{CP}$ for a wide range of objects. $\mathrm{R} \& \mathrm{~B}$ showed that this approach can produce $\mathrm{CP}$ in the case of an $\mathrm{e} / \mathrm{p}$ and also in the case of a (charge-asymmetric) $\mathrm{e}^{ \pm}$plasma.

However, in order to explain the long-term stability of the observed sign of $V$ it has to be assumed that the weak magnetic mean flux has to retain its polarity. This might surprise, since the dynamical time scales in several of the observed systems are orders of magnitudes smaller than the time-scale over which stability of the sign of $V$ could be established ${ }^{8}$. Furthermore, the large fluctuations in $V$ indicate strong temporal variations in the strength of the mean field. Since the latter should be dynamically unimportant, its stable polarity may be best explained if it results from an environmental large scale field, which gets dragged into the central engine. An alternative possibility was suggested by the anonymous referee: the mean field may be a remnant field of a stochastic superposition of many independent magnetic flux patches. The dynamical timescale for the evolution of the mean field could be on timescales much larger than the timescale of the evolution of an individual magnetic patch.

\subsection{Twisted magnetic fields}

The formalism of Sect. 2.1 can also be applied to the case of inhomogeneous magnetic fields if all coefficients in Eq. (1) become spatially dependent, and the FR coefficient is replaced by

$\kappa_{\mathrm{F}} \rightarrow \kappa_{\mathrm{R}}=\kappa_{\mathrm{F}}+\Omega . \quad$ Here, $\Omega=-2 \mathrm{~d} \phi_{B} / \mathrm{d} z$

describes the rate of linear polarisation rotation along the LOS due to the rotation of the coordinate frame, which was tied to the sky-projected magnetic fields for convenience. $\phi_{B}$ is the position angle of the sky-projected magnetic field in a fixed (non-varying) coordinate frame. For a purely stochastic magnetic field, $\Omega$ is a random variable with zero mean. However, if there is a systematic twist in the magnetic fields along the LOS $\Omega$ has a preferred sign. The sign of $\Omega$ does not depend on the polarity of the field, only on the handedness of the twist along the LOS. The effect of magnetic twist for CP production is mathematically identical to that of FR, but only if a

${ }^{8}$ E.g. SgrA* and GRS $1915+105$. SgrA* exhibited a stable $V$ sign on a timescale (20 years) which is 5 orders of magnitude longer than the dynamical timescale of the accretion flow close to the black hole $(\sim$ h). GRS $1915+105$ was observed to exhibit a LP-rotator event, but no change in the sign of CP (Fender et al. 2002, who described an indication of a CP sign reversal to be insignificant). However, counterexamples seem to exist, e.g. a CP sign reversal seems to be observed during the onset of a radiation outburst of GRO J1655-40 in 1994 (Macquart et al. 2002).

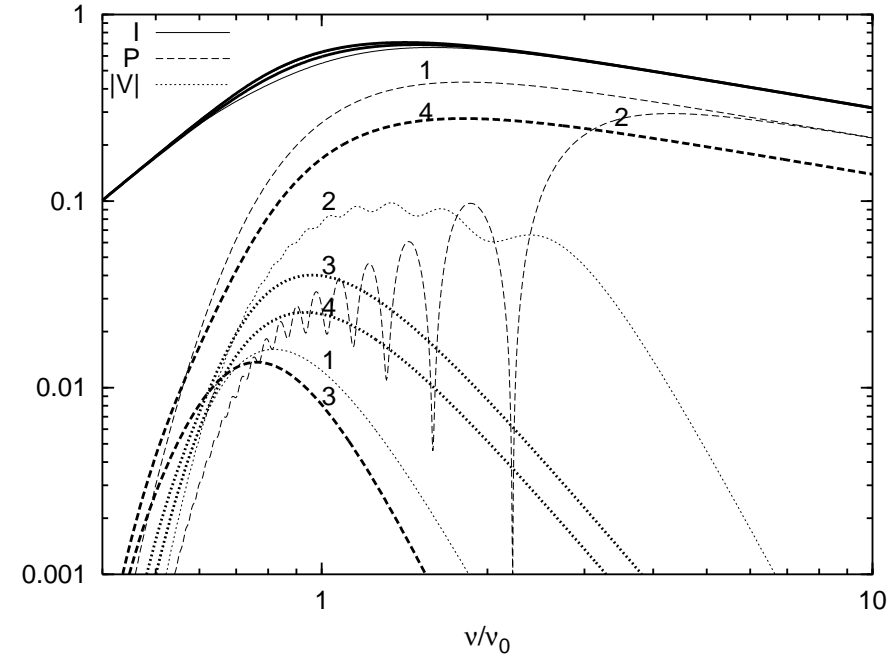

Fig. 1. Model spectra from numerical solutions of the radiative transfer along a LOS with homogeneous parameters. Shown is the total intensity $I$, the linearly polarised flux $P=\sqrt{Q^{2}+U^{2}}$, and the circular polarisation $|V|$. The sign of $V$ is always negative in the given examples, in accordance with the argumentation in the text. The LOS is characterised by $\varepsilon_{I}=\left(v / v_{0}\right)^{-\alpha}, \varepsilon_{Q}=(\alpha+1) /(\alpha+5 / 3) \varepsilon_{I}, \varepsilon_{U}=\varepsilon_{V}=0$, $\kappa_{I}=\left(v / v_{0}\right)^{-\alpha-5 / 2}, \kappa_{Q}=(\alpha+1) /(\alpha+5 / 3) \kappa_{I}, \kappa_{\mathrm{R}}=\tilde{\kappa}_{\mathrm{F}}\left(v / v_{0}\right)^{-2}+\Omega$, and $\kappa_{\mathrm{C}}=\tilde{\kappa}_{\mathrm{C}}\left(v / v_{0}\right)^{-3}$. The depth of the source is assumed to be $\Delta z=1$, so that $\tau=1$ for $v=v_{0}$. The thin lines (models $1 \& 2$ ) are FR based models, whereas the thick lines (models $3 \& 4$ ) are magnetic twist based models. The model parameters are $\alpha=0.5$ in all cases, $\tilde{\kappa}_{\mathrm{F}}=1,30,0,0$, $\Omega=0,0,2 \pi, \pi$, and $\tilde{\kappa}_{\mathrm{C}}=-0.3,-10,-1,-0.3$ respectively for model $1,2,3,4$. Note, that the parameters were chosen to illustrate the variety of possible spectra, to lead to an effective CP production, and to provide a readable figure. Therefore the parameters do not necessarily represent typical situations, and in some cases they are even counter examples to trends discussed in the text (e.g. model 2 exhibits strong $\mathrm{CP}$ due to an optimally chosen $\kappa_{\mathrm{C}}$ ).

single frequency is regarded, since FR is frequency dependent, whereas magnetic twist is not. However, CP production is possible in a completely non-Faraday rotating medium, e.g. as a charge symmetric $\mathrm{e}^{ \pm}$plasma. This is illustrated in Fig. 1, where simple model spectra for FR based and magnetic twist based $\mathrm{CP}$ generation scenarios are presented.

The requirement of $\tau_{\mathrm{R}}=\int \mathrm{d} z \kappa_{R} \sim 1$ for effective conversion translates into the requirement that the total magnetic twist along a LOS within the converting region is of the order of one (optimally it would be below $\pi / 2$ in order to avoid cancellation of $V$ contributions with opposite signs). It is argued below that this condition can be fulfilled naturally. In order to have magnetic twist being the dominant cause of $Q$ to $U$ conversion, FR has to be sufficiently suppressed. We assume in the following that $\tau_{\mathrm{F}} \ll 1$ holds within the optical thin part of the emission region, and show that this naturally leads to the observed sign persistence of $V$. The suppression of FR could be due to a vanishing leptonic charge density $\left(\rho_{\mathrm{e}}\right)$ as expected for a $\mathrm{e}^{ \pm}$plasma, or due to the presence of aligned, separate or nested flux tubes (with a global large scale twist) with independently oriented (but aligned) internal magnetic fields. As $\mathrm{B} \& \mathrm{~F}$ and $\mathrm{R} \& \mathrm{~B}$ showed for the stochastic field case, this can strongly reduce the Faraday depth also in the case of a large 


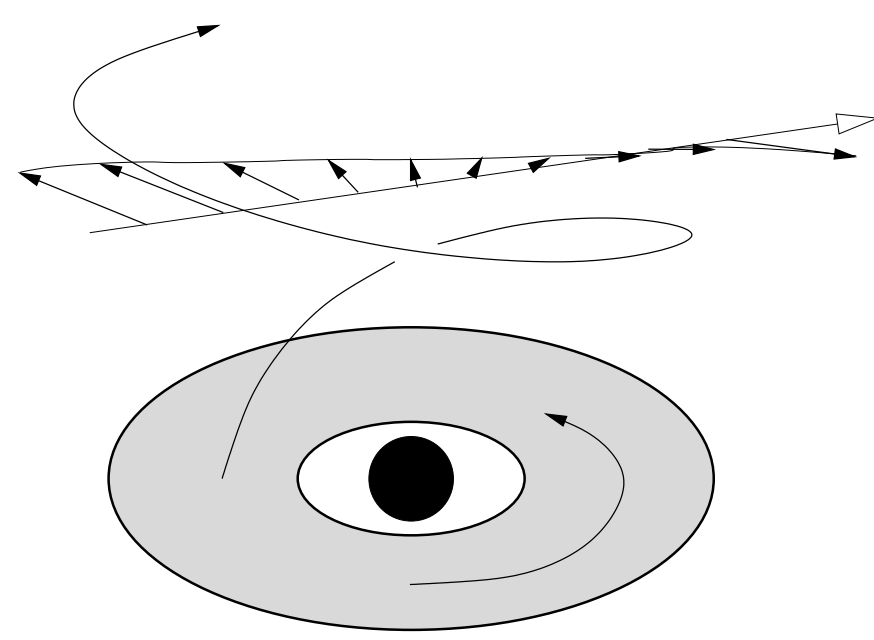

Fig. 2. Sketch of a possible geometry of a jet source. Shown is a positively rotating accretion disk around a central black hole from which a jet is launched (not shown). A single helical field line of the jet is drawn, and the magnetic field orientation along a typical LOS is indicated, demonstrating the presence of magnetic twist around the LOS.

microscopic $\kappa_{\mathrm{F}}$. In their cases, some fine-tuning was required since FR should be somehow suppressed, but not completely, leaving to sufficient FR from $Q$ to $U$. This fine-tuning is not required here, since the geometrical rotation provides $Q$ to $U$ rotation even in the case of fully suppressed FR. $\kappa_{\mathrm{C}}$ is not affected by LOS reversals of the magnetic field direction, since it only depends on the invariant $B_{y}^{2}$.

\section{Circular polarisation and rotating flows}

\subsection{A jet scenario}

Twisted magnetic fields are expected to be a natural ingredient of any quasar central engine due to the rotational nature of the accretion flow. A helically twisted magnetic field is expected to be present in jet outflows, especially if jets are magnetohydrodynamically launched (Blandford \& Payne 1982), but also otherwise, due to decreasing rotation speed of the sideways expanding outflow. The strength of the twisting depends on the ratio of the rotation to the outflow velocity (modulo beaming). In typical situations one would expect both velocities to be of the same order of magnitude, leading to $\left|\tau_{\mathrm{R}}\right| \sim 1$, as required for an efficient conversion in the absence of FR. Further, $\left|\tau_{R}\right| \leq 2 \pi$, where equality corresponds to a full reversal of the field across the jet in case of a completely wound up toroidal field. If the fields are very toroidal $\left(\tau_{\mathrm{R}} \geq \pi\right)$ an optical depth $\tau>1$ can help to increase $\mathrm{CP}$ by restricting the effective $\mathrm{FC}$ volume to a fraction of the LOS through the jet, and therefore suppressing CP cancellation effects caused by a production of $V$ of both signs.

A typical geometry is sketched in Fig. 2 for an approaching jet, which is rotating positively (in sky-projection). The rotation sense of the sky-projected magnetic fields seen by a photon flying from its emission point (where the synchrotron process provided it with $Q>0$ ) to the observer is therefore also positive, leading to $\Omega=-2 \mathrm{~d} \phi_{B} / \mathrm{d} z<0$ in this geometry. Thus, in our limit $\kappa_{\mathrm{F}} \ll \Omega$ we obtain $V \propto \Omega \kappa_{\mathrm{C}} Q \geq 0$ since $\kappa_{\mathrm{C}} \leq 0$ always.

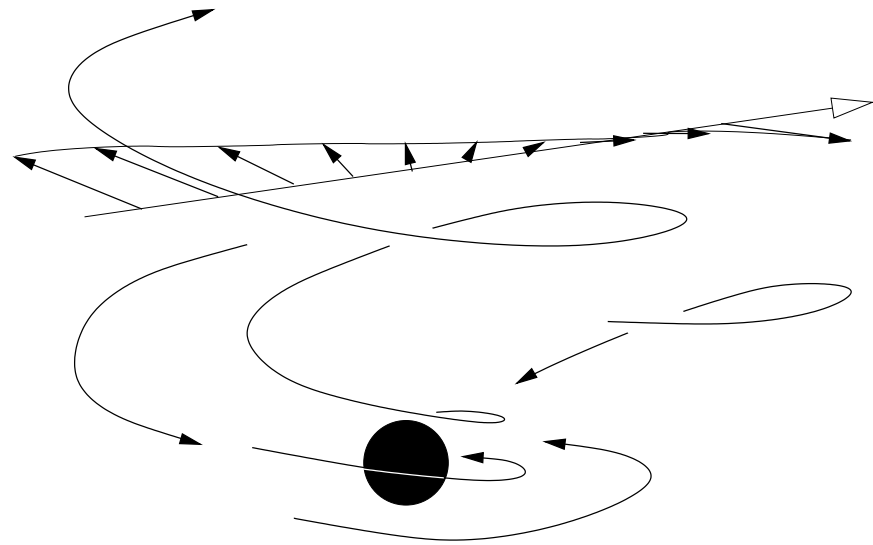

Fig. 3. Sketch of a possible ADAF onto a black hole. The flow direction and a single magnetic field line are shown. The converging and spiralling flow should imprint a helical twist into the magnetic field, which has exactly the same handedness as the one expected from a similarly rotating outflow, as shown in Fig. 2. The twist of the magnetic field along a LOS (also shown) has therefore the same sign in both models.

In this picture a positively rotating approaching jet emits negatively (clockwise) rotating circularly polarised light. Since the counter jets should exhibit an oppositely twisted magnetic field, it should produce $\mathrm{CP}$ of the opposite sign. However, the approaching jet dominates the total emission due to relativistic beaming so that its $\mathrm{CP}$ emission dominates the $\mathrm{CP}$ of both jets. We can conclude that the rotation of the received photons of a synchrotron emitting jet source are expected to be retrograde to - and therefore reveal - the sense of rotation of the central engine, which is the rotation of the accretion disk and/or the black hole.

\subsection{An ADAF scenario}

In an ADAF (Narayan \& Yi 1994) CP can also result from converted synchrotron emission, if the ADAF region produces synchrotron emission. The handedness of the magnetic twist is expected to be the same as the one in jets (if FR is suppressed in ADAFs) for the following reason: the ADAF is a converging flow, that is spinning up due to angular momentum conservation, whereas the jet is an expanding down-spinning flow. The way magnetic fields should be dragged in both cases has therefore the same handedness with respect to the spin axis of the system (see Fig. 3). This leads to the same handedness of the magnetic twist in the jet and ADAF case (on the upper and lower hemisphere of the black hole respectively). If the received flux is dominated by the upper (nearer) side of the ADAF, due to optical depth effects (CP sources are often selfabsorbed), or due to the black hole absorption of photons from the lower ADAF hemisphere, a net $\mathrm{CP}$ results, which counterrotates to the ADAF. The fractional $\mathrm{CP}$ of an ADAF may be comparable to that of a jet since $\left|\tau_{R}\right| \sim 1$ and $\left|\tau_{R}\right| \leq 2 \pi$ should hold for the same reasons as in the jet case. CP is therefore not necessarily uniquely a jet signature. 


\section{Discussion and outlook}

Twisted magnetic flux can produce circularly polarised synchrotron emission due to FC. If FR is sufficiently suppressed the expected sense of rotation of the emission is expected to be opposite to the one of the central engine, independent of the question whether the emission results form a synchrotron jet, or from a synchrotron emitting ADAF, and independent of the polarity of the magnetic fields. The FR suppression can be as discussed by $\mathrm{B} \& \mathrm{~F}$ and $\mathrm{R} \& \mathrm{~B}$ - due to a charge symmetric $\mathrm{e}^{ \pm}$plasma, in which FR is absent, or due to small-scale magnetic field polarity changes which produce cancelling FR contributions, but do not affect FC if the different flux tubes show alignment. Such polarity changes could be present if the magnetic field is organised in many helically aligned flux tubes of independent magnetic field polarity which originated in differently magnetised patches of the accretion disk.

This model is not in conflict with other proposed generation mechanisms for $\mathrm{CP}$ (as e.g. stochastic Faraday rotation with a small mean magnetic field as proposed by B\&F and R\&B), rather, it supplements them. Several of its strengths listed below are also present in other models. However, a few of the strengths (No. 1., 2., and 6.) seem to be slight advantages, potentially suggesting that it could be the dominant mechanism in several cases:

1. The sign of the CP does not depend on the presence of a mean field, but on geometrical properties of the flow pattern in the central engine of a quasar, which are fixed by the system's angular momentum. This may explain naturally the observed sign persistence of the $\mathrm{CP}$ over periods which exceed the dynamical timescales of the system by orders of magnitude.

2. The requirement that the rotation of the angle between LP and projected magnetic field is of the order one (not much higher, since over-rotation reduces $\mathrm{CP}$, not much lower in order to have FC operating) within the optically thin part of the emission region, is naturally fulfilled by the geometrical properties of jet and ADAF flows. This requirement implies some level of fine-tuning in other models where the rotation is due to FR.

3. CP is still converted LP in this scenario, so that the variability of CP should exceed the one of LP as observed.

4. The mechanism can work in jets and in ADAFs.

5. It is able to produce $\mathrm{CP}$ in an $\mathrm{e}^{ \pm}$and in an e/p plasma.

6. The relatively large robustness of the mechanism to variations in the underlying source properties - as composition of the Faraday converting plasma and nature of the emission region (jet or ADAF) - may explain why CP appears in a large variety of very different systems, such as microquasars, low luminosity AGNs, and powerful quasars.

7. In cases where CP exceeds LP (e.g. SgrA*), an outer Faraday screen, e.g. located in a mixing layer around an $\mathrm{e}^{ \pm}$jet, could provide the depolarisation (e.g. Homan et al. 2001). Alternatively, a $\pi / 2$ rotation of the sky-projected magnetic field throughout the visible emission region (the full optical thin jet, or the $\tau \leq 1$ region) could remove any observable LP, but leave a measurable amount of CP.
There are conditions, under which the here proposed relation between the (predominant) rotational sense of $\mathrm{CP}$ and the rotation of the central engine is not valid anymore. Whenever the average FR is stronger than the twist in the magnetic fields along the LOS, the sign of the average magnetic field will determine the sign of $\mathrm{CP}$ as in the models of $\mathrm{B} \& \mathrm{~F}$ and $\mathrm{R} \& \mathrm{~B}$. This may occur temporarily, if thermal plasma gets mixed into an $\mathrm{e}^{ \pm}$jet. If the emission region contains an e/p plasma, the occurrence of a mean magnetic field - either as a statistical fluctuation, or due to some physical reason - can increase the average Faraday depth and lead to a CP reversal. External CP generation from LP due to environmental scintillation also can mask the intrinsic CP signature of a quasar. Also within the scenario in which $\mathrm{CP}$ is due to bending in magnetic fields, CP sign reversals are possible, whenever the magnetic twist within the emission region is not linked to the central engine rotation. This may happen temporarily in violent shocks in a jet outflow, and would suggest that CP sign reversals of this origin are likely accompanying emission outbursts of the source ${ }^{9}$. The $\mathrm{CP}$ should also change if the rotational sense of the central engine is changed, e.g. due to a black hole merging event, or due to a massive infall of fresh material onto the accretion disc. Note, that the rarity of $\mathrm{CP}$ sign reversals indicate that the above discussed conditions should be exceptional, if CP is due to the here proposed mechanism.

The proposed mechanism provides testable predictions:

1. The sign of CP from a quasar measures the rotation direction of the quasar's engine. The electric vector of radio emission should rotate retrograde in the sky-plane with respect to the engine rotation. Positive $V$ polarisations implies therefore positive $=$ counter-clockwise rotation (on the sky) of the engine.

2. Thus, we expect SgrA*, which exhibited $V<0$ during the last 20 years, to rotate clockwise. This is retrograde with respect to the galactic rotation and the rotation of the molecular gas cloud in the galactic centre. However, it has the same rotation sense as the population of young hot HeI stars in its direct vicinity (Genzel et al. 2000), which were proposed to feed SgrA* via their stellar winds (Genzel 2001, for discussion and references). Conservation of angular momentum in this wind should lead to a retrograde (clockwise) rotating accretion disk. Alternatively, the spin of the central black hole may determine the twist of the magnetic fields in the emission region.

3. We expect the microquasars GRS $1915+105$ and SS 433 (both exhibit $V<0$ ) also to rotate clockwise, and we could give corresponding expectations for the other sources with detected CP.

4. The predominant sign of CP should be temporally constant. If the model is correct, quasar-like CP sources should only exhibit CP flips under exceptional circumstances, as discussed above.

5. CP from the counter jet should have opposite handedness than CP from the approaching jet. This prediction may allow a discrimination of the presented model from FR based

9 This may explain the observed CP sign reversal in GRO J1655-40 in during an outburst in 1994, as reported by Macquart et al. (2002). 
scenarios, since in the latter the same predominant CP sign is expected from both jets for a dipole-like mean field component.

6. There are possibly also spectral signatures, which allow to discriminate the different models. E.g. in FR based scenarios frequency dependent LP oscillations can occur, as illustrated by model 2 in Fig. 1, whereas this is impossible in the magnetic twist based scenarios. However, due to cancellation effects in the superposition of many slightly different LOSs such oscillations likely appear as a strong suppression of LP. Due to the high complexity of the resulting polarisation spectra general rules are not obvious and would require further investigations. In that context it is interesting to note that Beckert (in preparation) recently presented new model calculations for SgrA*. In order to simultaneously explain new high frequency LP measurements of SgrA* model parameters seem to be favoured for which magnetic twist is more important for $\mathrm{CP}$ production than FR.

If the scenario of $\mathrm{CP}$ production proposed here could be demonstrated to be operatively in quasars, it would give us a tool to measure the sense of rotation of the most powerful and enigmatic engines of the universe - the violent matter flows in the direct vicinity of black holes - by simply looking at the spin of the received photons.

Acknowledgements. I acknowledge useful discussions with F. Meyer, S. Heinz, H. Spruit, T. Beckert, H. Falcke, and C. Pfrommer. The manuscript benefited from several critical comments of an anonymous referee. After submission of this paper I became aware that on a recent conference (The Central 100 Parsecs, Hawaii, Nov. 3-8, 2002) T. Beckert presented new model calculations of the polarisation spectra of SgrA* in which a helical field geometry plays the the same role as in this work.

\section{References}

Beckert, T., \& Falcke, H. 2002, A\&A, 388, 1106

Björnsson, C.-I. 1990, MNRAS, 242, 158

Blandford, R. D., \& Payne, D. G. 1982, MNRAS, 199, 883

Bower, G. C., Falcke, H., \& Backer, D. C. 1999, ApJ, 523, L29

Bower, G. C., Falcke, H., \& Mellon, R. R. 2002a, ApJ, 578, L103

Bower, G. C., Falcke, H., Sault, R. J., \& Backer, D. C. 2002b, ApJ, 571,843

Brunthaler, A., Bower, G. C., Falcke, H., \& Mellon, R. R. 2001, ApJ, 560, L123

Fender, R., Rayner, D., Norris, R., Sault, R. J., \& Pooley, G. 2000, ApJ, 530, L29

Fender, R. P., Rayner, D., McCormick, D. G., et al. 2002, MNRAS, 336, 39

Genzel, R. 2001, in Dynamics of Star Clusters and the Milky Way, ASP Conf. Ser., 228, 291

Genzel, R., Pichon, C., Eckart, A., Gerhard, O. E., \& Ott, T. 2000, MNRAS, 317, 348

Hodge, P. E. 1982, ApJ, 263, 595

Homan, D. C., Attridge, J. M., \& Wardle, J. F. C. 2001, ApJ, 556, 113

Homan, D. C., \& Wardle, J. F. C. 1999, AJ, 118, 1942

Jones, T. W., \& O'Dell, S. L. 1977a, ApJ, 214, 522

Jones, T. W., \& O'Dell, S. L. 1977b, ApJ, 215, 236

Komesaroff, M. M., Roberts, J. A., Milne, D. K., Rayner, P. T., \& Cooke, D. J. 1984, MNRAS, 208, 409

Macquart, J. 2002, Publ. Astron. Soc. Austr., 19, 43

Macquart, J.-P., Wu, K., Sault, R. J., \& Hannikainen, D. C. 2002 , A\&A, 396, 615

Narayan, R., \& Yi, I. 1994, ApJ, 428, L13

Ruszkowski, M., \& Begelman, M. C. 2002, ApJ, 573, 485

Sault, R. J., \& Macquart, J.-P. 1999, ApJ, 526, L85

Valtaoja, E. 1984, Ap\&SS, 100, 227

Wardle, J. F. C., Homan, D. C., Ojha, R., \& Roberts, D. H. 1998, Nature, 395, 457 